\title{
Utilidad de la ecografía pulmonar en la unidad de medicina intensiva
}

\author{
M. Colmenero ${ }^{a, *}$, M. García-Delgado ${ }^{a}$, I. Navarrete ${ }^{a}$ y G. López-Milena ${ }^{b}$ \\ ${ }^{a}$ Unidad de Medicina Intensiva, Hospital General, Servicio de Cuidados Críticos y Urgencias, Hospital Universitario \\ Virgen de las Nieves, Granada, España \\ ${ }^{\mathrm{b} S}$ Servicio de Radiodiagnóstico, Hospital Universitario Virgen de las Nieves, Granada, España
}

Recibido el 22 de febrero de 2010; aceptado el 13 de abril de 2010

Disponible en Internet el 18 de mayo de 2010

\author{
PALABRAS CLAVE \\ Ecografía pulmonar; \\ Unidad de cuidados \\ intensivos; \\ Edema de pulmón; \\ Derrame pleural; \\ Neumotórax; \\ Consolidación \\ pulmonar
}

\begin{abstract}
Resumen
Tradicionalmente, el abordaje diagnóstico por la imagen del tórax en el paciente crítico se ha basado en la radiografía simple anteroposterior. Sin embargo, esta presenta grandes limitaciones en la precisión diagnóstica de la enfermedad pleuropulmonar. La introducción de la tomografía axial computarizada resolvió en gran medida este problema, pero con el doble inconveniente de las dosis de radiación y del inevitable traslado fuera de la unidad de cuidados intensivos. En este contexto, la ecografía pulmonar, gracias a su portabilidad, se convierte en una técnica alternativa en determinadas situaciones, con la ventaja de realizarse a la cabecera de los pacientes, ser fácilmente reproducible y no administrar radiaciones ionizantes.

En la ecografía pulmonar, las costillas, la columna vertebral y el aire del pulmón actúan como barreras para los ultrasonidos, y provocan artefactos que debemos reconocer e interpretar para un correcto diagnóstico. No obstante, las enfermedades intratorácicas y la existencia de líquido en el espacio pleural, así como la consolidación o atelectasia en el pulmón proporcionan suficiente ventana ecográfica para una correcta evaluación.

En la siguiente revisión abordaremos los aspectos principales de la realización de la técnica de la ecografía pulmonar, definiremos el patrón normal y los artefactos que sirven de base para la detección de anormalidades, y explicaremos los criterios de los patrones de las principales enfermedades (consolidación, derrame pleural, edema de pulmón y neumotórax). También discutiremos la posible utilidad y las limitaciones que la ecografía pulmonar puede tener en diferentes situaciones de nuestra práctica clínica, como en el diagnóstico de la insuficiencia respiratoria aguda; la detección, la cuantificación y el drenaje del derrame pleural; el traumatismo torácico; las complicaciones y el tratamiento del síndrome de distrés respiratorio agudo, y la comprobación de una correcta intubación traqueal.
\end{abstract}

(c) 2010 Elsevier España, S.L. y SEMICYUC. Todos los derechos reservados.

\footnotetext{
*Autor para correspondencia.

Correo electrónico: macol@telefonica.net (M. Colmenero). 


\section{KEYWORDS}

Lung ultrasound; Intensive care unit; Pulmonary edema; Pleural effusion; Pneumothorax; Pulmonary consolidation

\section{Utility of the lung ultrasound in the intensive medicine unit}

\begin{abstract}
The radiological diagnostic approach of the thorax in the critically ill patient has traditionally been based on the anteroposterior chest X-ray. However, it is generally accepted that it has important limitations regarding its diagnostic accuracy of pleuropulmonary disease. The introduction of computed tomography largely solved this problem, but with the dual disadvantage of a larger radiation dose and the unavoidable transportation outside of the ICU. In this context, the lung ultrasound has become an alternative technique, with the advantage that due to its portability, it is done at the patient's bedside.

In the lung ultrasound, the ribs, spine and air in the thorax act as barriers to the ultrasounds, causing artifacts that must be recognized and interpreted for a correct diagnosis. However, intrathoracic diseases, existence of fluid in the pleural space and consolidation, or atelectasis in the lung provide a sufficient ultrasound window for the correct evaluation.

In this review, we explain the lung and pleural ultrasound technique, define the normal pattern and the artifacts that serve to detect the abnormalities and we explain the criteria for the main diseases (consolidation, pleural effusion, pulmonary edema and pneumothorax). We also discuss the possible utility and limitations of the lung ultrasound in our daily practice, such as diagnosis of acute respiratory failure, detection, quantification and drainage of a pleural effusion, chest trauma, management and complications of acute respiratory distress syndrome and tracheal intubation success or failure.
\end{abstract}

(c) 2010 Elsevier España, S.L. and SEMICYUC. All rights reserved.

\section{Introducción}

La ecografía se ha revelado como una técnica de imagen de gran utilidad en los pacientes críticos ${ }^{1}$. Dos son las características que la han convertido en un procedimiento de primera línea en la exploración de estos enfermos graves. La primera es la ausencia de emisión de radiaciones, lo que la hace especialmente apropiada para determinados grupos (niños, mujeres embarazadas) y además permite la repetición de la prueba tantas veces como sea necesaria, sin incrementar el riesgo. La segunda ventaja es la portabilidad a la cabecera del paciente, aspecto especialmente importante por lo que supone de evitación de riesgos en los traslados ${ }^{2}$.

De todas las áreas de exploración, las que se consideran esenciales en el currículo de los intensivistas $\operatorname{son}^{3}$ la ecocardiografía (básica y avanzada), la ecografía vascular, tanto para la cateterización venosa central como para la detección de trombosis venosa, y la ecografía pulmonar (denominada por algunos grupos pleuropulmonar). En el caso de los traumatizados, se ha desarrollado una combinación simple de varias de estas denominada focused assesment with sonography for trauma ${ }^{4}$ (FAST).

Tradicionalmente, el abordaje diagnóstico del tórax en el paciente crítico se ha basado en la radiografía simple anteroposterior, que es esencial en la evaluación de los múltiples dispositivos asociados al tratamiento de estos pacientes (tubo traqueal, catéteres venosos centrales, etc.), pero que presenta grandes limitaciones en la precisión diagnóstica de la enfermedad pleuropulmonar ${ }^{5}$. La introducción de la tomografía axial computarizada (TAC) resolvió en gran medida este problema con una resolución cada vez mejor, pero con el inconveniente de las dosis de radiación y, sobre todo, el inevitable traslado fuera de la unidad de cuidados intensivos $^{6}$. En este contexto aparece la ecografía pulmonar que, liderada por los propios intensivistas, se ha ido desarrollando progresivamente tanto en sus aspectos conceptuales como de aplicación práctica a la cabecera de los pacientes.

En la siguiente revisión abordaremos los aspectos principales de la realización de la técnica de la ecografía pulmonar, definiremos el patrón normal y los artefactos que sirven de base para la detección de anormalidades, y los patrones de las principales enfermedades (consolidación, derrame pleural, edema de pulmón y neumotórax). También discutiremos la posible utilidad y las limitaciones que la ecografía pulmonar puede tener en diferentes situaciones de nuestra práctica clínica, como el diagnóstico de la insuficiencia respiratoria aguda; la detección, cuantificación y drenaje del derrame pleural; el traumatismo torácico; las complicaciones y el tratamiento del síndrome de distrés respiratorio agudo, y la comprobación de una correcta intubación traqueal.

\section{Técnica de examen}

El equipo de ecografía necesario para la realización de la técnica solo precisa de imagen bidimensional y en modo $M$, pero actualmente casi todos los equipos modernos están dotados de otras posibilidades (Doppler, color, armónicos, etc.). Más importante es la disponibilidad de sondas multifrecuencia o de varios tipos de sonda. Las sondas de frecuencias entre $3,5-5 \mathrm{MHz}$, utilizadas para la exploración abdominal y cardiaca, proporcionan una adecuada 


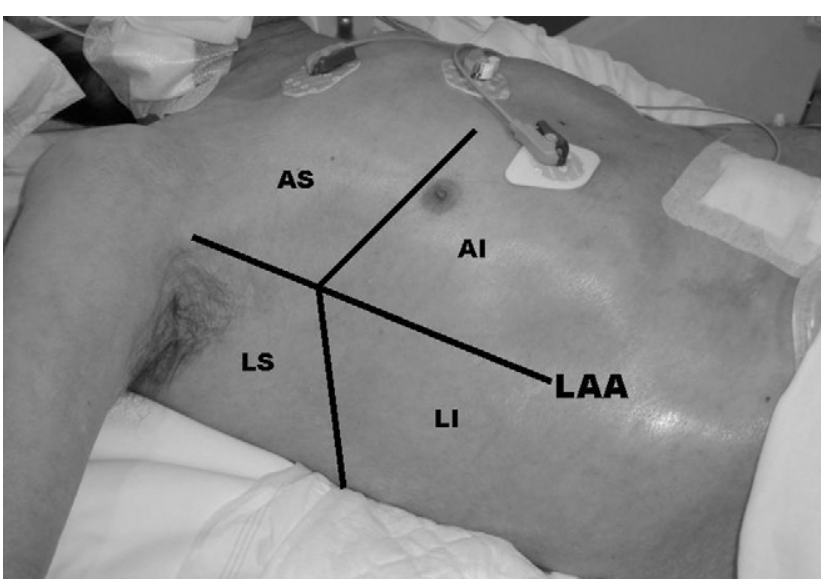

Figura 1 Áreas de exploración. Al: anteroinferior; AS: anterosuperior; LAA: línea axilar anterior; LI: lateroinferior LS: laterosuperior.

visualización de los planos profundos, y permiten la caracterización de las consolidaciones y el derrame pleural. Para las estructuras más superficiales, es decir, la pleura y los signos y los artefactos que se generan de ella, las sondas de frecuencia superior a $5 \mathrm{MHz}$ proporcionan mucha mejor resolución. Estas sondas se emplean, además, para el estudio vascular, lo que nos sirve de complemento en el diagnóstico de la trombosis venosa profunda. La forma de la sonda utilizada (lineal, convexa o sectorial) también dependerá de la zona a estudiar, y será convexa si el espacio intercostal es estrecho, para evitar la interferencia acústica que producen las costillas.

Los transductores sectoriales no son, en general, adecuados para el examen del espacio pleural por vía intercostal directa, ya que presenta una visión muy estrecha en los campos cercanos y el espacio pleural se identifica mal por los artefactos.

En los pacientes críticos, la exploración se realiza en la posición de decúbito supino, que permite un fácil abordaje anterolateral. Cada grupo de investigadores ha desarrollado una sistemática de exploración propia, a veces excesivamente prolija y compleja ${ }^{7}$. Nosotros nos alineamos junto con los que establecen que son suficientes 4 áreas en cada hemitórax, y que lo dividen en 2 zonas, anterior y lateral, con la línea axilar anterior como divisoria ${ }^{8}$. A su vez, cada zona se divide en una superior y otra inferior según una línea horizontal que atravesaría la unión del tercio medio con el inferior del esternón (fig. 1). La exploración comenzaría por la zona anterosuperior y acabaría por la lateroinferior, con cortes longitudinales y transversales. A veces es necesario el estudio de las zonas dorsales, para lo que es precisa una ligera inclinación del enfermo, que se logra exclusivamente con una leve aducción del brazo ipsolateral. Aunque algunos expertos afirman que puede hacerse una exploración completa en unos $3 \mathrm{~min}$, nuestra experiencia es que son necesarios entre 5-10 minutos, según la complejidad de los hallazgos.

\section{Patrones ecográficos: imagen normal}

Al situar el transductor lineal en sentido longitudinal perpendicular a los espacios intercostales se visualizan cerca de los

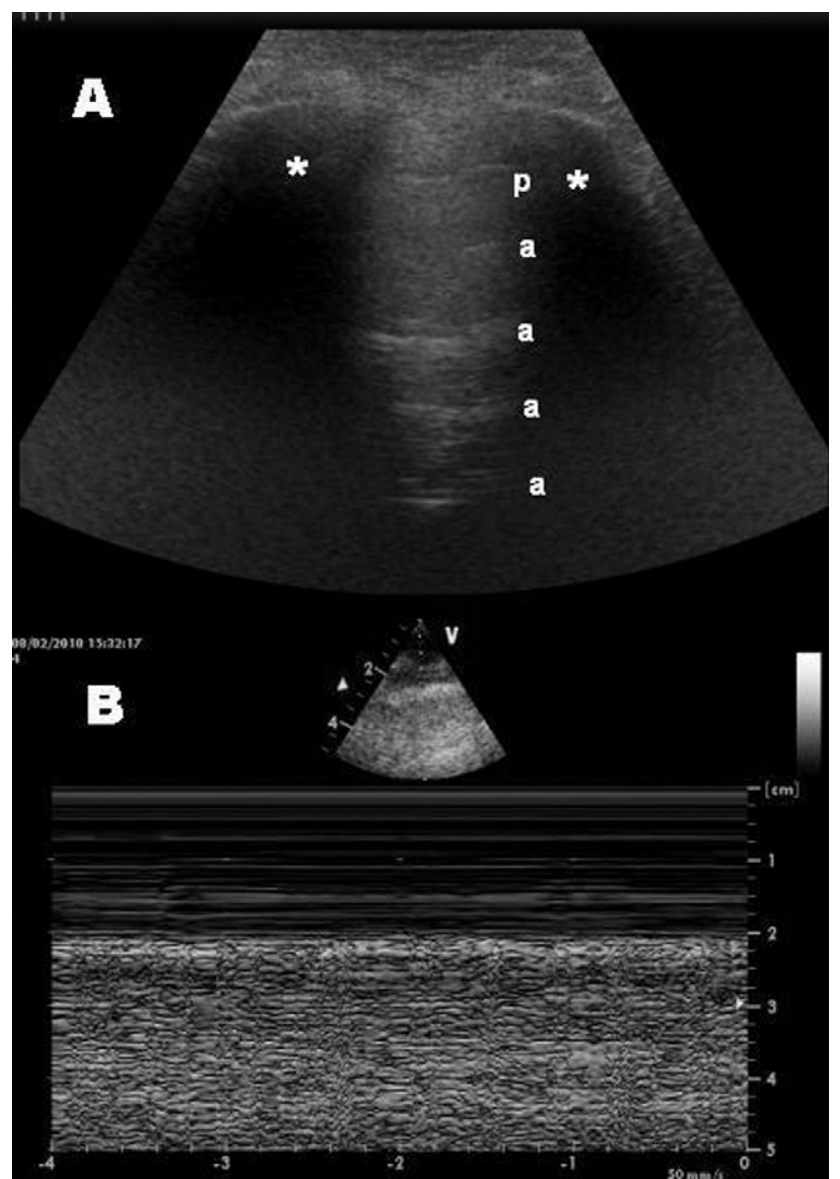

Figura 2 Imagen normal. A) Modo bidimensional: signo del murciélago. B) Modo $\mathrm{M}$ : signo de la orilla de playa. *: sombra costal; a: líneas A; p: línea pleural.

bordes laterales de la pantalla 2 interfases ecogénicas redondeadas con una marcada sombra acústica que corresponde a las costillas (fig. 2). Aproximadamente a medio centímetro por debajo del inicio de estas se sitúa una línea hiperecogénica horizontal que representa la pleura. Con transductores de alta frecuencia y gran resolución a poca profundidad se pueden observar las 2 capas, parietal y pleural, de unos $2 \mathrm{~mm}$ de anchura y de aspecto regular, separadas entre sí por el espacio pleural $(0,3 \mathrm{~mm})$. Sin embargo, con los transductores que se emplean habitualmente (de $<5 \mathrm{MHz}$ ), la línea es única y representa la superficie de unión pleuropulmonar. En conjunto, el borde superior de las sombras de las costillas y la línea pleural componen una imagen que semeja el perfil de un murciélago, y por esto ha recibido la denominación bat sign.

El pulmón aireado subyacente a la pleura es una interfase altamente reflectante que bloquea la penetración de los haces de ultrasonidos. El patrón del pulmón normal representa, por tanto, un artefacto por reverberación con múltiples ecos de una intensidad media, de aspecto moteado. La principal característica que hay que observar es la presencia de «deslizamiento» o «signo del planeo» en la superficie de unión pleuropulmonar, que ocurre obligatoriamente con la insuflación y el vaciado pulmonar durante las fases respiratorias. Si aplicamos el modo $M$, se distinguen 2 zonas bien diferenciadas, que configuran el signo de la orilla (seashore sign): la parte superior, que corresponde a la 
pared torácica, formada por líneas horizontales paralelas (el mar), y la parte inferior, desde la pleura, de aspecto granulado, como arena de playa (sandy beach).

También en modo real (bidimensional) se puede visualizar el movimiento de ambos hemidiafragmas y cómo en inspiración el pulmón se desplaza caudalmente, y aparece en ocasiones como un velo o una cortina.

\section{Artefactos}

Las imágenes que no se corresponden con ninguna estructura fácilmente reconocible, sino que se producen por parte de la reflexión y la reverberación de los ecos sobre las interfases de los tejidos, se denominan artefactos. En ecografía existen múltiples artefactos derivados de las propiedades del haz de ultrasonido asociados a errores en la velocidad por la atenuación de los diferentes tejidos 9 . En la ultrasonografía pulmonar los más importantes se visualizan como una serie de líneas, horizontales o verticales, y hay que conocer lo que representan y en qué entidades se producen, pero a su vez hay que diferenciarlas entre sí para evitar errores de interpretación.

Estas líneas principalmente se deben a artefactos de reverberación que se producen cuando la señal de ultrasonido se refleja de forma repetida entre interfases altamente reflectoras que están cerca del transductor ${ }^{10}$.

Las más importantes son las siguientes:

- Líneas A: líneas hiperecogénicas horizontales y paralelas que se sitúan a una distancia múltiplo de la que existe entre el transductor y la línea pleural.

- Líneas B o cola de cometa (comet tail artifact): líneas hiperecogénicas verticales que parten desde la pleura y alcanzan el final de la pantalla. Si son múltiples se denominan patrón B o cohetes (lung rockets). Como veremos, son expresión de edema o fibrosis de los septos interlobulares.

- Líneas C: líneas hiperecogénicas horizontales que se sitúan a una distancia que no es múltiplo de la que existe entre el transductor y la línea pleural.

- Líneas E: líneas hiperecogénicas verticales que se inician en la pared torácica (a diferencia de las líneas B que parten de la línea pleural). Se producen con enfisema subcutáneo.

- Líneas Z: líneas hiperecogénicas verticales que se inician en la pleura pero que no alcanzan el final de la pantalla (lo que las diferencia de las líneas B).

En la tabla 1 se describen los principales signos en ecografía pulmonar, tanto en condiciones de normalidad como asociados a procesos patológicos.

\section{Síndrome alveolointersticial}

La presencia de edema en el tejido pulmonar se manifiesta por el hallazgo de líneas B o imágenes en cola de cometa (fig. 3). Estas líneas deben reunir las siguientes características: partir de la línea pleural, alcanzar el borde inferior de la pantalla, borrar las líneas A y desplazarse al son de los movimientos respiratorios ${ }^{11}$. Todas estas propiedades las distinguen de las líneas $Z$ y las líneas $E$, de distinta significación. El mecanismo
Tabla 1 Signos en ecografía pulmonar

Signo del deslizamiento pleural (gliding o sliding sign): imagen en modo real del movimiento de la pleura visceral (que acompaña al pulmón) sobre la parietal

Signo de la orilla (seashore sign): en modo $M$ se distinguen 2 zonas bien diferenciadas. La parte superior, que corresponde a la pared torácica, formada por líneas horizontales paralelas. La parte inferior, desde la pleura, de aspecto granulado, como arena de playa (sandy beach)

Signo de la medusa (jellyfish sign) o de la lengua (tonguelike sign): se corresponde con una atelectasia completa del lóbulo pulmonar (generalmente inferior) que «flota» sobre un derrame pleural masivo

Signo del sinusoide (sinusoid sign): movimiento centrífugo (hacia el transductor) de la línea que representa la interfase, generalmente la pleura visceral, con la inspiración

Signo del plancton: imágenes puntiformes hiperecogénicas móviles en el seno de un derrame pleural. Son indicativas de un exudado o hemotórax

Signo del punto pulmonar (lung point sign): en modo $M$ se produce una sucesión de imágenes normales (arenosas) durante la inspiración y anormales (líneas horizontales) durante la espiración. Es el punto del tórax en el que en inspiración el pulmón «toca» o alcanza a la pared torácica en el seno de un neumotórax no masivo

Signo de la estratosfera (stratospfere sign) o del código de barras (bar code sign): en modo M solo se visualizan líneas horizontales paralelas

Signo del límite regular (quad sign): de los bordes bien definidos (regulares). Se produce en el derrame pleural, al estar siempre localizado entre ambas pleuras

Signo del límite irregular (shred sign, signo de la scie o dientes de sierra): de las tiras o bordes irregulares. Se produce en la consolidación, generalmente neumónica, y el borde más profundo está sin definir, salvo que afecte a todo un lóbulo

Signo del latido pulmonar (lung pulse): en modo $\mathrm{M}$ el aspecto granulado se intensifica con líneas verticales a intervalos coincidentes con el latido cardiaco. Representa un paso intermedio hacia la atelectasia completa, como sucede en los primeros momentos tras una intubación selectiva

Signo del artefacto pulmonar (cardiac-lung): en pacientes en ventilación mecánica se observa en la ventana apical de la ecocardiografía transtorácica una «masa» intracardiaca, que en modo $M$ presenta un patrón respirofásico similar al signo del sinusoide. Se trata de una imagen especular de una consolidación pulmonar o derrame pleural

de producción de estas líneas se debe a la reverberación de los haces de ultrasonido al reflejarse sobre los septos interlobulares engrosados.

Según la distancia en la pleura entre las líneas B se puede diferenciar la localización del edema. Así, las líneas separadas entre sí alrededor de $7 \mathrm{~mm}$ se corresponden con edema intersticial, mientras que las que se distancian $3 \mathrm{~mm}$ indican la presencia de edema alveolar.

La ecografía no distingue la naturaleza del fluido que se acumula (agua, pus) o del tejido que prolifera (fibrótico, infiltrativo), ni la del mecanismo que produce el paso desde 


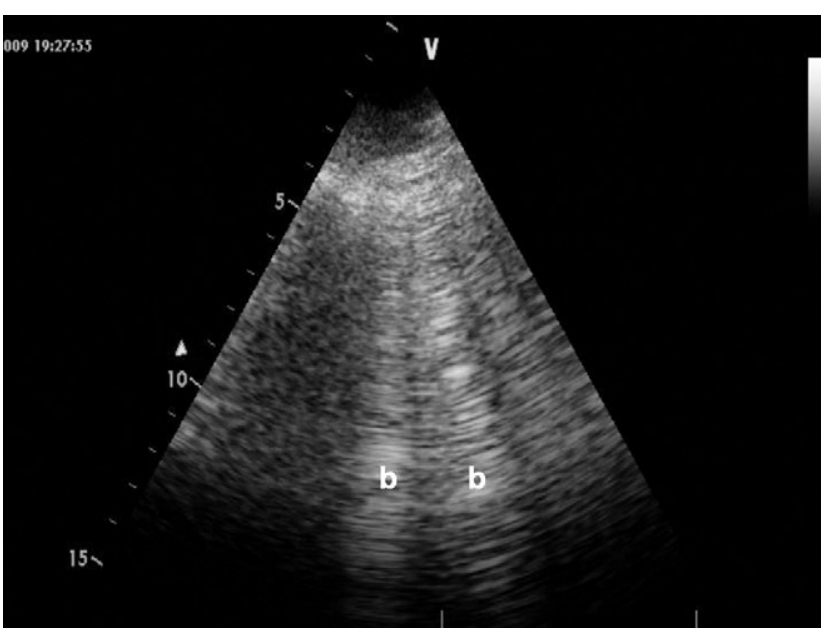

Figura 3 Síndrome alveolointersticial. b: líneas b.

el vaso al intersticio (hidrostático o por aumento de la permeabilidad). La diferenciación entre las entidades que producen este síndrome debe hacerse considerando otras características. Además del contexto clínico y los factores predisponentes y precipitantes, se han descrito algunos signos ecográficos que pueden ayudar en el diagnóstico diferencial ${ }^{12}$. Entre los que se consideran específicos del edema por aumento de la permeabilidad se encuentran las denominadas zonas parcheadas (áreas de pulmón normales junto a las patológicas), la ausencia de deslizamiento pleuropulmonar y la presencia de consolidaciones.

Algunos autores han tratado de relacionar la intensidad del síndrome alveolointersticial con la cuantía del edema pulmonar, y han utilizado como referencia la medición del agua pulmonar extravascular (APE). Así, Agricola et al emplean un sistema de puntuación (score) ultrasonográfico que consiste en la suma del total de líneas B observadas en el conjunto de zonas escaneadas (para estos autores, 28) ${ }^{13}$. Esta puntuación se correlaciona moderadamente con el APE medida por el método de termodilución transpulmonar. En la práctica clínica es útil considerar la puntuación como una estimación semicuantitativa del edema pulmonar. En función de la puntuación obtenida, catalogaríamos el edema como ausente ( $<5$ líneas $B$ ), leve (de 5-15), moderado (de 15-30) o grave (más de 30 ). La utilización de la ecografía puede resultar especialmente útil en aquellos casos sin expresividad radiológica por no haber alcanzado aún el umbral del edema alveolar, que suele producirse con un incremento del $50-75 \%$ del valor superior de la normalidad del APE. En este escenario, la presencia de un patrón ecográfico intersticial puede revelar la causa de la hipoxemia o poner de manifiesto el edema antes, incluso, de que se produzca un deterioro en el intercambio gaseoso ${ }^{14}$.

Además, la ecografía se ha revelado muy útil en la monitorización de la evolución de los pacientes con edema pulmonar y en la respuesta al tratamiento. Fagenholtz et al comprobaron en pacientes con edema pulmonar de las alturas cómo la mejoría clínica y en la saturación arterial de oxígeno se correlacionaba con la disminución en el número de líneas B en la ecografía pulmonar. Los autores hacen notar lo valiosa que es una herramienta diagnóstica de este tipo (portátil, ligera) en un área en la que no es posible disponer de los equipamientos hospitalarios clásicos ${ }^{15}$. Por su parte, Noble et al estudiaron a un grupo de pacientes con insuficiencia renal y sobrecarga hídrica, antes y después de la realización de hemodiálisis, y encontraron una asociación significativa entre el volumen de líquido retirado y la disminución en el número total de líneas $\mathrm{B}(2,7$ líneas por cada $500 \mathrm{ml})^{16}$.

\section{Consolidación pulmonar}

Para poder diagnosticar una consolidación pulmonar previamente se deben dar 2 requisitos. El primero es su localización intratorácica. Para esto en las zonas basales es importante localizar el diafragma, ya que en supino y con pérdida de volumen, tanto el hígado como el bazo pueden estar localizados más cranealmente y, por su aspecto, confundirse con una consolidación. El segundo es que exista contacto con la pleura para que los ultrasonidos la «vean». Esta condición se cumple en la inmensa mayoría de las consolidaciones $(90 \%)$, pero a veces hay que ser exhaustivo en la exploración de todas las áreas, especialmente las dorsales, para que no pasen desapercibidas.

En la consolidación pulmonar los espacios aéreos están rellenos de líquido o células inflamatorias, por lo que, en esta situación, el pulmón aireado que es altamente reflexógeno se convierte en una masa sólida, densa y con buena transmisión sónica.

El pulmón consolidado es hipoecogénico en comparación con el pulmón aireado y también, generalmente, con respecto al hígado y al bazo por su alto contenido acuoso.

Los criterios diagnósticos de la presencia de una consolidación pulmonar (fig. 4) son ${ }^{17-19}$ los siguientes:

- Patrón «tisular»: similar al del tejido de órganos sólidos como el hígado. Es el único criterio imprescindible, el resto se consideran adicionales.

- Límites anatómicos: mientras que el borde superficial correspondiente a la pleura es regular, el borde profundo se muestra irregular, salvo que exista una afectación lobar completa o sea una pequeña consolidación subpleural.

- Ausencia de artefactos (líneas A o B): el reemplazo de aire por otro tipo de material (edema, celularidad) o su

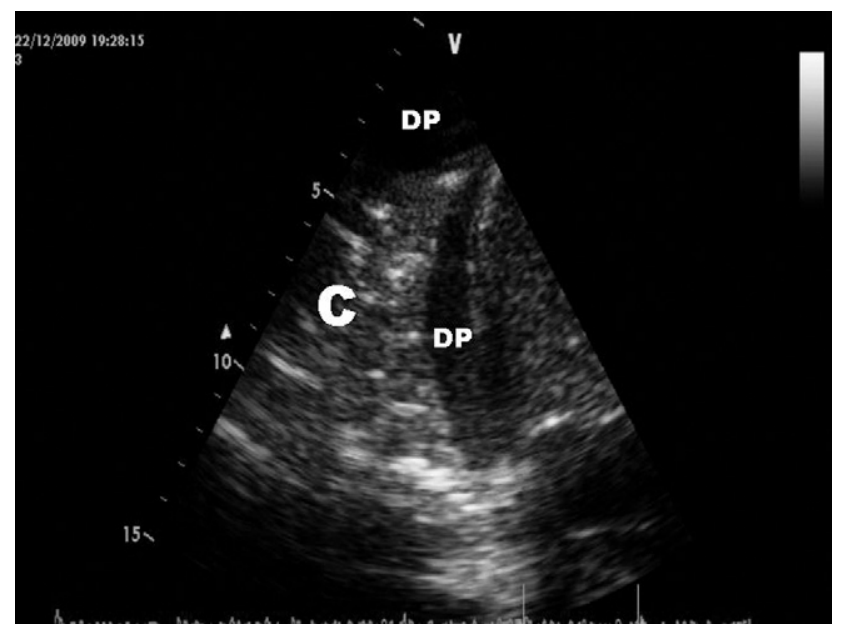

Figura 4 Imagen de consolidación pulmonar. C: consolidación; DP: derrame pleural. 
ausencia (colapso) impide la formación de los artefactos que dependen de la interacción en la interfase aire/fluido.

- Ausencia del «signo del sinusoide», lo que la distingue de un derrame pleural en aquellos casos en los que presenta una ecogenicidad reducida.

- Presencia de «broncograma aéreo» y «alveolograma aéreo»: la existencia de aire en las vías aéreas se manifiesta como imágenes hiperecogénicas puntiformes o lineales, en función de que se «corten» transversal u horizontalmente. Representan el aire en el interior de los bronquios o alvéolos rodeados del pulmón consolidado.

- Visualización de arterias y venas pulmonares intraparenquimatosas.

La consolidación puede ser una atelectasia (obstructiva o no obstructiva), neumonía, contusión, infarto, tumor, etc. La ecografía no distingue la naturaleza de la consolidación, por lo que son otros criterios clínicos u otras pruebas complementarias los que orientarán el diagnóstico. No obstante, empiezan a aparecer trabajos que tratan de encontrar criterios discriminativos entre unas y otras. Así, recientemente, el grupo de Lichtenstein ha comunicado que la presencia de broncograma aéreo dinámico puede servir para el diagnóstico diferencial entre la neumonía y la atelectasia por reabsorción ${ }^{20}$. Este signo se basa en el cambio que experimentan en la longitud $(>1 \mathrm{~cm})$ las líneas hiperecogénicas con los movimientos respiratorios, pero para esto es preciso no modificar el plano de exploración. Otros autores han descrito, con el Doppler color, irregularidades en el patrón vascular cuando se trata de neumonías.

\section{Neumotórax}

La detección de neumotórax es especialmente importante en el traumatismo torácico, el barotrauma asociado a la ventilación mecánica, tras la cateterización venosa central yugular o subclavia, y en pacientes a los que se va a trasladar en medios aéreos.

La ecografía es muy sensible para el diagnóstico del neumotórax incluso de pequeño tamaño (ocultos en la radiografía de tórax) ${ }^{21}$. Diferentes estudios la cifran entre un 90-100\%. Existen varios signos ecográficos. El primero es la ausencia de «deslizamiento pulmonar», ya que la pleura visceral pierde el contacto con la pleura parietal ${ }^{22}$. En el modo M se pierde la apariencia de «orilla de playa» (con arena y mar) y solo se visualizan líneas horizontales paralelas, imagen conocida como el signo de la estratosfera o código de barras (fig. 5A). No obstante, la falta de deslizamiento pulmonar puede producirse en otras enfermedades y circunstancias, como las adherencias pleurales, la intubación bronquial selectiva y la contusión, la consolidación y la atelectasia pulmonares ${ }^{23}$. Esta falta de especificidad hace que la decisión de realizar un tratamiento descompresivo inmediato basado exclusivamente en este signo dependa de las circunstancias clínicas del paciente. En el caso de inestabilidad hemodinámica (incluida la parada cardiocirculatoria), se optaría por la colocación de un drenaje. En el caso de que no existiera compromiso vital urgente, sería recomendable la realización de otra técnica de imagen.

La presencia de líneas B nos permite descartar un neumotórax, ya que implica la aposición de ambas pleuras, lo que permite que se forme el artefacto ${ }^{24}$.

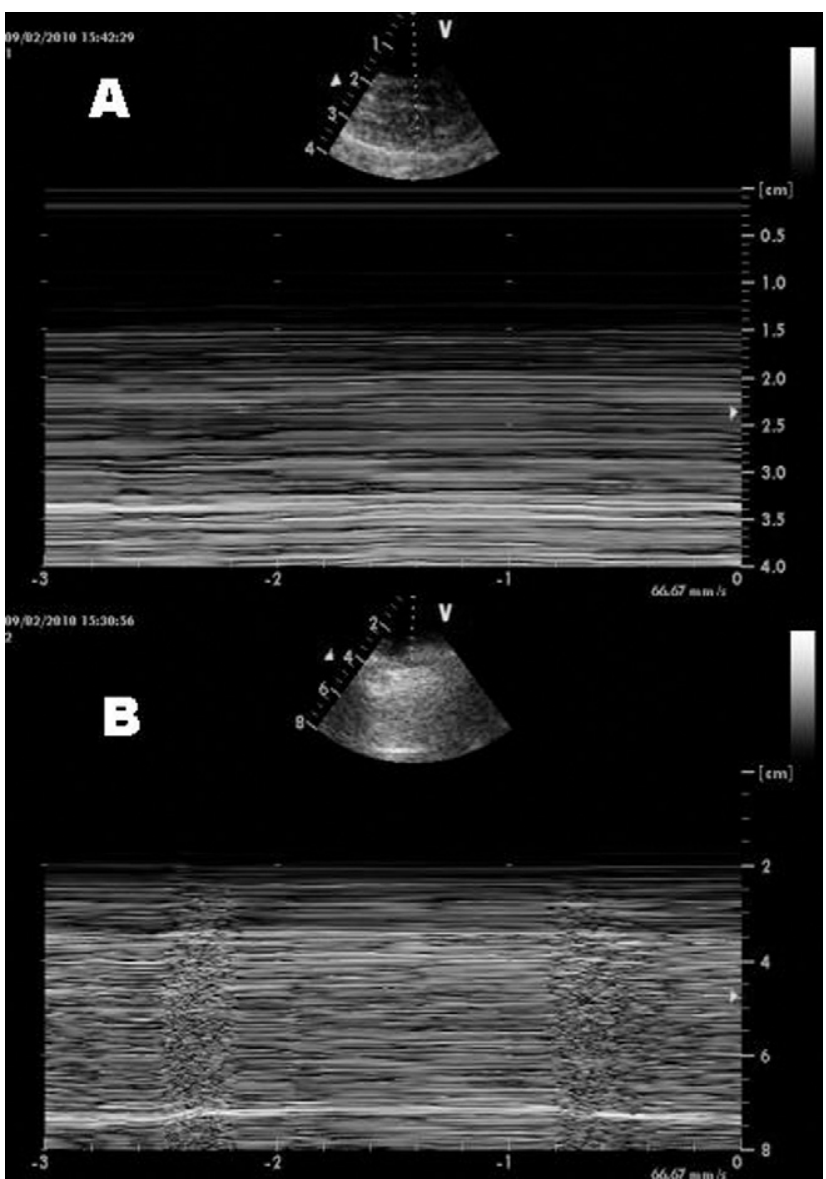

Figura 5 Imagen de neumotórax en modo M. A) Signo de la estratosfera. B) Signo del «punto pulmonar».

Por último, existe un signo específico que es el hallazgo del denominado «punto pulmonar» (lung point) en modo $M$ (fig. 5B). Se caracteriza porque se produce una sucesión de imágenes normales (arenosas) durante la inspiración y anormales (líneas horizontales) durante la espiración, y se corresponde con el punto del tórax donde, en inspiración, el pulmón «toca» o alcanza a la pared torácica en el seno de un neumotórax no masivo.

\section{Derrame pleural}

Mediante la aplicación de transductores lineales directamente sobre el tórax, el derrame pleural se visualiza como un espacio libre de ecos (imagen anecoica, «negra») entre la pleura parietal y la visceral ${ }^{25}$. Debe asegurarse que se encuentre localizado por encima del diafragma. El segundo signo definitorio procede de la imagen en modo $M$ a través del derrame, en el que se aprecian variaciones del espacio interpleural con el ciclo respiratorio, que disminuyen con la inspiración (o la insuflación del respirador). Es lo que se denomina signo del sinusoide (fig. 6A). Se puede acompañar de atelectasias compresivas en derrames cuantiosos, que se identifican como imágenes móviles de aspecto de medusa o lengua (fig. 6B).

También es frecuente detectar derrames pleurales con transductores sectoriales de $3,5 \mathrm{MHz}$ que utilizan como 


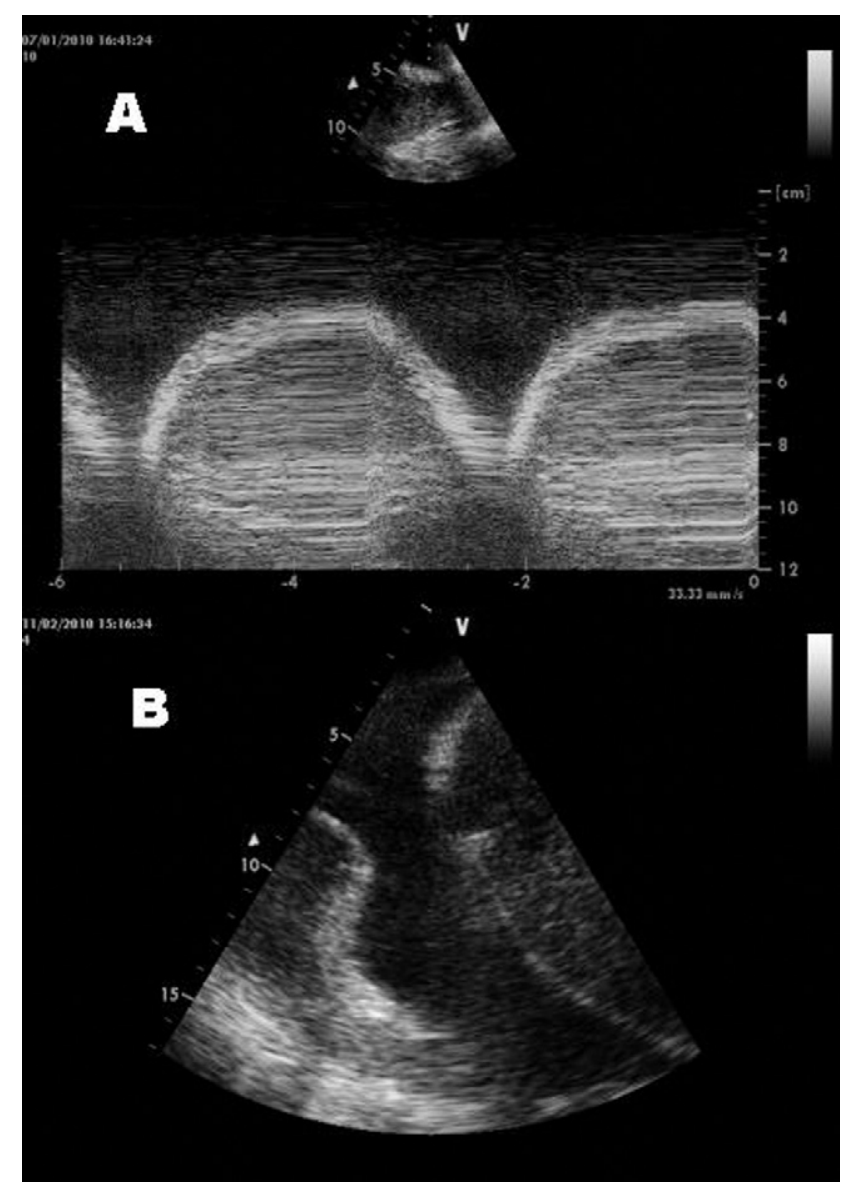

Figura 6 Imagen de derrame pleural. A) Signo del sinusoide. B) Signo de la medusa.

ventana el abdomen, donde se observaría el líquido hipoecogénico por encima del diafragma, visualización del interior del tórax a través de la colección y la inversión del diafragma si existieran grandes cantidades de líquido.

De acuerdo con la apariencia ecográfica, el derrame pleural se clasifica en los siguientes tipos:

- Simple: totalmente anecoico.

- Complejo no tabicado (sin septos): si existe material ecogénico en el seno de un derrame anecoico, generalmente puntiforme.

- Complejo tabicado (septado): si el material ecogénico tiene forma de filamentos o tabiques.

- Ecogénico difuso: por su apariencia con ecogenicidad aumentada homogéneamente, que se corresponde con la presencia de tejido desvitalizado, proteínas, fibrina y sangre.

Aunque la clasificación de un derrame en exudado o trasudado es a veces difícil, incluso con criterios de laboratorio, la distinción es útil en términos de actitud diagnóstico-terapéutica. Las características del derrame en la ecografía pueden ayudar a diferenciar uno de otro ${ }^{26}$. En general, los trasudados son anecoicos, pero un derrame anecoico puede ser cualquiera de los 2. Sin embargo, un derrame complejo (tabicado o no) y uno ecogénico difusamente son siempre exudados. Estos últimos se corresponden habitualmente con hemotórax o empiemas.
Otros signos que apoyan la naturaleza de exudado del derrame son la presencia de una consolidación, el engrosamiento y la existencia de nódulos pleurales.

De forma semicuantitativa, el volumen del derrame se puede clasificar como mínimo, pequeño, moderado o masivo en función del número de espacios intercostales en los que se visualiza. No obstante, en pacientes en ventilación mecánica, al estar en decúbito supino, si el derrame no está tabicado, se moviliza libremente a través del espacio pleural y se distribuye en función del grado de elevación de la cabecera de la cama. Esto puede modificar las medidas ecográficas del derrame, por lo que es preciso estandarizarlas. En general, se acepta una posición entre $0-15^{\circ}$, se explora en la línea axilar posterior tanto en corte longitudinal como transversal y se realiza la medida en la fase espiratoria. Existen varios estudios a este respecto, que, además, tratan de correlacionar las medidas del derrame con el volumen drenado. Roch et $\mathrm{al}^{27}$ midieron 2 longitudes, una fue la distancia entre la base del pulmón y el diafragma en un corte longitudinal, y otra la distancia interpleural entre el pulmón y la pared costal en un corte transversal en la zona basal. Este plano se referencia a $3 \mathrm{~cm}$ por encima de la base del pulmón en el corte longitudinal. Encontraron que esta última se correlacionaba mejor con el volumen del derrame $(r=0,68 ; p<0,001)$ y que una distancia superior a $5 \mathrm{~cm}$ predecía con una buena exactitud $(86 \%)$ un derrame de más de $500 \mathrm{ml}$. Vignon et $\mathrm{al}^{28}$, al referenciar el nivel basal al primer espacio intercostal en el que desaparece la visualización del diafragma, encontraron que una medida similar de la distancia interpleural máxima $(4,5 \mathrm{~cm}$ en el hemitórax derecho y $5 \mathrm{~cm}$ en el izquierdo) predecía un volumen superior a los $800 \mathrm{ml}$. Balik et $\mathrm{al}^{29}$ desarrollaron una fórmula simple en la que el volumen estimado (en mililitros) resultaba de multiplicar la distancia interpleural máxima (en milímetros) por 20.

\section{Aplicaciones clínicas}

La ecografía pulmonar, aislada o combinada con otras técnicas ecográficas (ecocardiografía, ecografía vascular) se emplea actualmente en las siguientes situaciones clínicas. Algunas de ellas se han consolidado en la práctica clínica y otras están en fase de investigación y requieren más estudios para confirmar su validez y su utilidad.

\section{Diagnóstico de la insuficiencia respiratoria aguda}

De acuerdo con un protocolo al que han denominado Bedside Lung Ultrasound in Emergency (BLUE), el grupo de Lichtenstein ha alcanzado una precisión diagnóstica del 90,5\% sobre 260 pacientes ingresados en una unidad de cuidados intensivos por insuficiencia respiratoria aguda ${ }^{30}$. Este protocolo consiste en la determinación de una serie de «patrones» ecográficos. Estos patrones se basan en la combinación del análisis del sistema venoso junto con la presencia o la ausencia de los siguientes signos: deslizamiento pleural, líneas A o B y derrame o consolidación. El diagnóstico diferencial incluyó las entidades más frecuentes (el 97,5\% de todos los casos), que fueron asma o agudización de la enfermedad pulmonar obstructiva crónica, edema de pulmón, embolia pulmonar, neumotórax y neumonía. Junto 
con la elevada exactitud diagnóstica, los autores destacan la precocidad en obtener un diagnóstico definitivo, que en este tipo de enfermos es de vital importancia.

Además de este protocolo global, la ecografía se ha utilizado para el diagnóstico de entidades concretas en el ámbito de las urgencias, tales como la neumonía radiooculta ${ }^{31}$ y la embolia pulmonar periférica ${ }^{32}$. En pacientes en ventilación mecánica ha puesto de manifiesto abscesos en el seno de neumonías con respuesta desfavorable al tratamiento ${ }^{33}$.

\section{Realización de técnicas/procedimientos}

- Toracocentesis y drenaje pleural: la evacuación de derrames con fines diagnósticos o terapéuticos fue uno de los primeros usos de la ecografía torácica ${ }^{34}$. Pronto se comprobó el aumento en la seguridad de la punción que proporcionaban la correcta y precisa localización y extensión del derrame ${ }^{35}$. En pacientes en ventilación mecánica se ha comprobado que reduce la incidencia de complicaciones, y se ha referido una tasa de neumotórax de tan solo el $1,3 \%^{36}$.

- Intubación traqueal: la ecografía pulmonar permite el diagnóstico de la intubación bronquial selectiva, ya que el pulmón no ventilado mostrará ausencia del signo de deslizamiento pulmonar ${ }^{37}$.

\section{Ampliación de la ecografía en el traumatismo grave (extended focused assesment with sonography for trauma)}

La clásica exploración abdominal para la detección del líquido libre peritoneal y pericárdico (subcostal) en pacientes traumatizados se puede complementar de forma rápida mediante la colocación de la sonda en la pared anterior y lateral del tórax con el fin de descartar neumotórax ${ }^{38}$. Su especificidad es muy alta y su sensibilidad es mayor que la de la radiografía, pero, sobre todo, se realiza como una extensión de la exploración física y el diagnóstico se realiza en un tiempo sensiblemente inferior a esta ${ }^{39}$. En este sentido, su principal utilidad radicaría en determinar durante los primeros minutos de la evaluación del paciente traumatizado si es necesaria la colocación de un tubo torácico. Por otra parte, aunque puede detectar más neumotórax ocultos, la decisión sobre su tratamiento debe incluir una valoración de la estabilidad cardiorrespiratoria del paciente y de los procedimientos que se le van a realizar (ventilación mecánica, transporte intercentro o aéreo $)^{40}$.

Aunque no explícitamente incluida en el extended focused assesment with sonography for trauma, en el entorno del paciente traumatizado también se ha utilizado la ecografía para la detección de la contusión pulmonar ${ }^{41}$. Parece especialmente relevante en los primeros estadios, cuando no presenta expresividad radiológica ni clínica, pero su hallazgo puede predecir una mala evolución.

\section{Monitorización de la respuesta a los tratamientos en pacientes críticos}

- Administración de fluidoterapia: tanto en pacientes sépticos como en afectados de lesión pulmonar y distrés respiratorio agudo, la administración de fluidoterapia debe hacerse de forma muy juiciosa. Se ha demostrado tanto el beneficio de una reanimación inicial precoz y generosa como el efecto pronóstico adverso que existe con el balance acumulativo positivo de fluidos, tanto en los pulmones como general. La presencia de un patrón con líneas A (ausencia de edema) permitiría continuar con la fluidoterapia en la reanimación, al ser el factor limitante de la administración de más volumen la aparición de líneas B. Más complejas serían la restricción hídrica o la inducción de diuresis en los pacientes en los que ya existe edema, en los que hay que considerar conjuntamente los parámetros hemodinámicos que garanticen la perfusión tisular ${ }^{42}$.

- Tratamiento antibiótico en la neumonía: recientemente el grupo de la Pitié-Salpêtrière, en París, ha publicado los resultados del uso de la ecografía pulmonar en la evaluación de la respuesta al tratamiento antibiótico en la neumonía asociada a ventilación mecánica ${ }^{43}$. Para esto realizaron un seguimiento de un grupo de pacientes durante 7 días con tratamiento antibiótico y compararon con los resultados de la TAC. La resolución de la neumonía se categorizó en función del cambio en el patrón ecográfico de menor a mayor aireación (consolidación, alveolar, intersticial, normal). La correlación con la TAC fue excelente.

- Reclutamiento: con una metodología similar a la anterior, Gardelli et $\mathrm{al}^{44}$ han publicado la relación entre los diferentes patrones ecográficos de «apertura» pulmonar y el resultado de las maniobras de selección. Aunque no específicamente estudiada todavía, la distribución de los infiltrados en el SDRA (patrón focal o difuso) y su respuesta a la presión positiva (potencial de reclutamiento) podrían evaluarse con la ecografía y ayudar en la elección de la mejor estrategia ventilatoria ${ }^{45}$.

Como conclusión podemos afirmar que la ecografía pulmonar, antaño considerada como un «imposible», se está consolidando como una herramienta diagnóstica de gran versatilidad y utilidad para el tratamiento de los pacientes críticos, tanto en las áreas de urgencias como en las unidades de medicina intensiva ${ }^{46,47}$. Para una correcta utilización de esta es imprescincible una formación reglada y el conocimiento de sus limitaciones.

\section{Bibliografía}

1. Liechtenstein D. Ultrasound diagnosis of the critically ill, 2 ed. Berlin-Heidelberg: Springer-Verlag; 2005.

2. Beckmann U, Gillies DM, Berenholtz SM, Wu AW, Pronovost P. Incidents relating to the intrahospital transfer of critically ill patients. An analysis of the reports submitted to the Australian Incident Monitoring Study in Intensive Care. Intensive Care Med. 2004;30:1579-85.

3. Mayo PH, Beaulieu Y, Doelken P, Feller-Kopman D, Harrod C, Kaplan A. American College of Chest Physicians/La Societé de Réanimation de Langue Française. Statement on Competence in Critical Care Ultrasonography. Chest. 2009;135:1050-60.

4. Bahner D, Blaivas M, Cohen HL, Fox JC, Hoffenberg S, Kendall J, et al. AIUM practice guideline for the performance of the focused assessment with sonography for trauma (FAST) examination. J Ultrasound Med. 2008;27:313-8. 
5. Bekemeyer WB, Crapo RO, Calhoon S, Cannon CY, Clayton PD. Efficacy of chest radiography in a respiratory intensive care unit. A prospective study. Chest. 1985;88:691-6.

6. Beckmann U, Gillies DM, Berenholtz SM, Wu AW, Pronovost P. Incidents relating to the intrahospital transfer of critically ill patients. An analysis of the reports submitted to the Australian Incident Monitoring Study in Intensive Care. Intensive Care Med. 2004;30:1579-85.

7. Jambrik Z, Monti S, Coppola V, Agricola E, Mottola G, Miniati M, et al. Usefulness of ultrasound lung comets as a nonradiologic sign of extravascular lung water. Am J Cardiol. 2004;93:1265-70.

8. Volpicelli G, Mussa A, Garofalo G, Cardinale L, Casoli G, Perotto F, et al. Bedside lung ultrasound in the assessment of alveolarinterstitial syndrome. Am J Emerg Med. 2006;24:689-96.

9. Merrit CRB. Física de los ultrasonidos. En: Rumack CM, Wilson SR, Charboneau JW, Johnson JAM, editores. Diagnóstico por ecografía, 3 ed. Madrid: Mosby; 2006:3-34.

10. Feldman MK, Katyal S, Blackwood MS. US artifacts. Radiographics. 2009;29:1179-89.

11. Lichtenstein D, Meziere G, Biderman P, Gepner A, Barre O. The comet-tail artifact. An ultrasound sign of alveolar-interstitial syndrome. Am J Respir Crit Care Med. 1997;156:1640-6.

12. Copetti R, Soldati G, Copetti P. Chest sonography: A useful tool to differentiate acute cardiogenic pulmonary edema from acute respiratory distress syndrome. Cardiovasc Ultrasound. 2008;6:16-26.

13. Agricola E, Bove T, Oppizi M, Marino G, Zangrillo A, Margonato A, et al. "Ultrasound comet-tail images": A marker of pulmonary edema: A comparative study with wedge pressure and extravascular lung water. Chest. 2005;127:1690-5.

14. Gargani L, Lionetti V, Di Cristofano C, Bevilacqua G, Recchia FA, Picano E. Early detection of acute lung injury uncoupled to hypoxemia in pigs using ultrasound lung comets. Crit Care Med. 2007;35:2769-74.

15. Fagenholz PJ, Gutman JA, Murray AF, Noble VE, Thomas SH, Harris NS. Chest ultrasonography for the diagnosis and monitoring of high-altitude pulmonary edema. Chest. 2007;131:1013-8.

16. Noble VE, Murray AF, Capp R, Sylvia-Reardon MH, Steele DJ, Liteplo A. Ultrasound assessment for extravascular lung water in patients undergoing hemodialysis. Time course for resolution. Chest. 2009;135:1433-9.

17. Yang PC, Luh KT, Chang DB, Yu CJ, Kuo SH, Wu HD. Ultrasonographic evaluation of pulmonary consolidation. Am Rev Respir Dis. 1992;146:757-62.

18. Targhetta R, Chavagneux R, Bourgeois JM, Dauzat M, Balmes P, Pourcelot L. Sonographic approach to diagnosing pulmonary consolidation. J Ultrasound Med. 1992;11:667-72.

19. William E, Brant MD. Tórax. En: Rumack CM, Wilson SR, Charboneau JW, Johnson JAM, editores. Diagnóstico por ecografía, 3 ed. Madrid: Mosby; 2006:575-97.

20. Lichtenstein D, Mezière G, Seitz J. The dynamic air bronchogram: A lung ultrasound sign of alveolar consolidation ruling out atelectasis. Chest. 2009;135:1421-5.

21. Chung MJ, Goo JM, Im JG, Cho JM, Cho SB, Kim SJ. Value of highresolution ultrasound in detecting a pneumothorax. Eur Radiol. 2005;15:930-5.

22. Lichtenstein D, Menu Y. A bedside ultrasound sign ruling out pneumothorax in the critically ill: Lung sliding. Chest. 1995; 108:1345-8.

23. Murphy M, Nagdev A, Sisson C. Lack of lung sliding on ultrasound does not always indicate a pneumothorax. Resuscitation. 2008;77:270.

24. Lichtenstein DA, Mezière G, Biderman P, Gepner A. The comettail artifact: An ultrasound sign ruling out pneumothorax. Intensive Care Med. 1999;25:383-8.

25. Eibenberger KL, Dock WI, Ammann ME, Dorffner R, Hormann MF, Grabenwoger F. Quantification of pleural effusions: Sonography versus radiography. Radiology. 1994;191:681-4.
26. Yang PC, Luh KT, Chang DB, Wu HD, Yu CJ, Kuo SH. Value of sonography in determining the nature of pleural effusion: Analysis of 320 cases. Am J Roentgenol. 1992;159:29-33.

27. Roch A, Bojan M, Michelet P, Romain F, Bregeon F, Papazian L, et al. Chest. 2005;127:224-32.

28. Vignon P, Chastagner C, Berkane V, Chardac E, François B, Normand $\mathrm{S}$, et al. Quantitative assessment of pleural effusion in critically ill patients by means of ultrasonography. Crit Care Med. 2005;33:1757-63.

29. Balik M, Plasil P, Waldauf P, Pazout J, Fric M, Otahal M, et al. Ultrasound estimation of volume of pleural fluid in mechanically ventilated patients. Intensive Care Med. 2006;32:318-21.

30. Lichtenstein DA, Mezière G. Relevance of lung ultrasound in the diagnosis of acute respiratory failure: The BLUE protocol. Chest. 2008;134:117-25.

31. Volpicelli G, Caramello V, Cardinale L, Cravino M. Diagnosis of radio-occult pulmonary conditions by real-time chest ultrasonography in patients with pleuritic pain. Ultrasound Med Biol. 2008;34:1717-23.

32. Mathis G, Blank W, Reissig A, Lechleitner P, Reuss J, Schuler A, et al. Thoracic ultrasound for diagnosing pulmonary embolism: A prospective multicenter study of 352 patients. Chest. 2005;128:1531-8.

33. Lichtenstein DA, Peyrouset 0 . Is lung ultrasound superior to CT? The example of a CT occult necrotizing pneumonia Intensive Care Med. 2006;32:333-5.

34. Yu CJ, Yang PC, Chang DB. Diagnostic and therapeutic use of chest sonography: Value in critically ill patients. AJR Am J Roentgenol. 1992;159:695-701.

35. Lichtenstein D, Hulot JS, Rabiller A, Tostivint I, Mezière G. Feasibility and safety of ultrasound-aided thoracocentesis in mechanically ventilated patients. Intensive Care Med. 1999;25: 955-8.

36. Mayo PH, Goltz HR, Tafreshi M, Doelken P. Safety of ultrasoundguided thoracentesis in patients receiving mechanical ventilation. Chest. 2004;125:1059-62.

37. Weaver B, Lyon M, Blaivas M. Confirmation of endotracheal tube placement after intubation using the ultrasound sliding lung sign. Acad Emerg Med. 2006;13:239-44.

38. Kirkpatrick AW, Sirois M, Laupland KB, Liu D, Rowan K, Bal CG, et al. Hand- held thoracic sonography for detecting posttraumatic pneumothoraces: The extended focused assessment with sonography for trauma (EFAST). J Trauma. 2004;57:288-295.

39. Zhang M, Liu ZH, Yang JX, Gan JX, Xu SW, You XD, et al. Rapid detection of pneumothorax by ultrasonography in patients with multiple trauma. Crit Care. 2006;10:R112.

40. Ball CG, Kirkpatrick AW, Feliciano DV. The occult pneumothorax: What have we learned? Can J Surg. 2009;52:173-9.

41. Soldati G, Testa A, Silva FR, Carbone L, Portale G, Silveri NG. Chest ultrasonography in lung contusion. Chest. 2006;130:533-8.

42. Lichtenstein DA, Mezière GA, Lagoueyte JF, Biderman $P$, Goldstein I, Gepner A. A-lines and B-lines: Lung ultrasound as a bedside tool for predicting pulmonary artery occlusion pressure in the critically ill. Chest. 2009;136:1014-20.

43. Bouhemad B, Liu ZH, Arbelot C, Zhang M, Ferarri F, Le-Guen M, et al. Ultrasound assessment of antibiotic-induced pulmonary reaeration in ventilator-associated pneumonia*. Crit Care Med. 2010;38:84-92.

44. Gardelli G, Feletti F, Gamberini E, Bonarelli S, Nanni A, Mughetti M. Using sonography to assess lung recruitment in patients with acute respiratory distress syndrome. Emerg Radiol. 2009;16:219-21.

45. Arbelot C, Ferrari F, Bouhemad B, Rouby JJ. Lung ultrasound in acute respiratory distress syndrome and acute lung injury. Curr Opin Crit Care. 2008;14:70-4.

46. Vollmer I, Gayete A. Ecografía torácica. Arch Bronconeumol. 2010;46:27-34.

47. Bouhemad B, Zhang M, Lu Q, Rouby JJ. Clinical review: Bedside lung ultrasound in critical care practice. Critical Care. 2007; 11:205. 